Honam Mathematical J. 34 (2012), No. 3, pp. 375-379

http://dx.doi.org/10.5831/HMJ.2012.34.3.375

\title{
ON A SYMMETRIC FUNCTIONAL EQUATION
}

\author{
Jae-Young Chung
}

\begin{abstract}
We find a general solution $f: G \rightarrow G$ of the symmetric functional equation$$
x+f(y+f(x))=y+f(x+f(y)), \quad f(0)=0
$$

where $G$ is a 2-divisible abelian group. We also prove that there exists no measurable solution $f: \mathbb{R} \rightarrow \mathbb{R}$ of the equation. We also find the continuous solutions $f: \mathbb{C} \rightarrow \mathbb{C}$ of the equation.
\end{abstract}

\section{Introduction}

In [3], Marcin E. Kuczma introduced the functional equation

$$
x+g(y+f(x))=y+g(x+f(y))
$$

which arises while studying a problem of compatibility of means. In particular, he obtained analytic solutions $f, g: \mathbb{R} \rightarrow \mathbb{R}$ of the equation (1.1). In [2], Nicole Brillouët-Belluot refined the result and find the differentiable solution of the equation (1.1). In the paper, it is also proved that the functional equation

$$
x+f(y+f(x))=y+f(x+f(y)), f(0)=0
$$

has no differentiable solution $f: \mathbb{R} \rightarrow \mathbb{R}$. In the present paper we prove, with a different approach from those in [2], that there exist no measurable solutions $f: \mathbb{R} \rightarrow \mathbb{R}$ of the equation (1.2). As a matter of fact we find a general solution of the equation (1.2) for the function

Received June 12, 2012. Accepted July 1, 2012.

2000 Mathematics Subject Classification. 39B82.

Key words and phrases. symmetric functional equation, additive function, measurable function. 
$f: G \rightarrow G$ when $G$ is a 2 -divisible abelian group. As a consequence we find a continuous solution $f: \mathbb{C} \rightarrow \mathbb{C}$ of the equation (1.2).

\section{Main theorems}

We denote by $G$ a 2-divisible abelian group with identity 0 and $f$ : $G \rightarrow G$. We first consider the functional equation

$$
x+f(y+f(x))=y+f(x+f(y)), \quad f(0)=0
$$

for all $x \in G$. We say that $f$ is additive provided that

$$
f(x+y)=f(x)+f(y)
$$

for all $x, y \in G$.

Theorem 2.1. Let $f$ satisfy (2.1). Then $f$ is an additive function satisfying

$$
f(f(x))=f(x)-x
$$

for all $x, y \in G$. Conversely, if an additive function $f$ satisfies (2.3), then $f$ satisfies (2.1).

Proof. Letting $y=0$ in (2.1) we get (2.3). Replacing $x$ by $f(x)$ in (2.3) we have

$$
f(f(f(x)))=f(f(x))-f(x)=-x
$$

Replacing $x$ by $f(f(x))$ in (2.1) and using (2.4) and (2.3) we have

$$
f(f(y)+f(f(x)))=f(f(x))+f(y-x)-y=f(x)+f(y-x)-x-y
$$

Replacing $x$ by $f(x)$ and $y$ by $f(y)$ in (2.1) we have

$$
f(x)+f(f(y)+f(f(x)))=f(y)+f(f(x)+f(f(y))) .
$$

From (2.5) and (2.6) we have

$$
2 f(x)+f(y-x)=2 f(y)+f(x-y) .
$$


Letting $y=0$ in $(2.7)$ we have $f(-x)=-f(x)$. Thus, replacing $x-y$ by $u$ and $y$ by $v$ we have

$$
2 f(u+v)=2 f(u)+2 f(v),
$$

which implies that $f$ is additive since $G$ is 2-divisible. Conversely, assume that $f$ satisfies (2.2) and (2.3). Then we have

$$
\begin{aligned}
& x+f(y+f(x))=x+f(y)+f(f(x))=f(y)+f(x), \\
& y+f(x+f(y))=y+f(x)+f(f(y))=f(x)+f(y) .
\end{aligned}
$$

Also, from (2.2) we have $f(0)=0$. This completes the proof.

From the above result it is easy to see that there exist no regular solutions $f: \mathbb{R} \rightarrow \mathbb{R}$ of (2.1).

Corollary 2.2. The functional equation (2.1) has no solution $f$ : $\mathbb{R} \rightarrow \mathbb{R}$ which is bounded in a set of positive measure.

Proof. Let $f: \mathbb{R} \rightarrow \mathbb{R}$ satisfy (2.1). Then by Theorem $2.1, f$ satisfies (2.2) and (2.3). It is well known that every solution $f: \mathbb{R} \rightarrow \mathbb{R}$ of (2.2), which is bounded in a set of positive measure, has the form $f(x)=a x$ for some $a \in \mathbb{R}([1])$. By (2.3) we have $a^{2}=a-1$. Thus $a$ is not a real number. This completes the proof.

Remark. The Corollary 2.2 implies there are no measurable (continuous, increasing, and so on) function $f: \mathbb{R} \rightarrow \mathbb{R}$ satisfying (2.1).

Now we find a continuous solution $f: \mathbb{C} \rightarrow \mathbb{C}$ of the equation (2.1).

Corollary 2.3. The continuous solution $f: \mathbb{C} \rightarrow \mathbb{C}$ of the equation (2.1) has the form

$$
f(z)=\left(\frac{1}{2} \pm i \sqrt{\frac{3}{4}+|\beta|^{2}}\right) z+\beta \bar{z}
$$

for some constant $\beta \in \mathbb{C}$. 
Proof. Let $f(z)=g(z)+i h(z), g(z), h(z) \in \mathbb{R}$ satisfy (2.1). Then by Theorem 2.1, both $g$ and $h$ are additive since $f$ is additive. Let $z=$ $x+i y, x, y \in \mathbb{R}$ and $g_{1}(x)=g(x), g_{2}(y)=g(i y), h_{1}(x)=h(x), h_{2}(y)=$ $h(i y)$. Then $g_{1}, g_{2}, h_{1}, h_{2}$ are all continuous additive function. By the well known fact([1]), we obtain

$$
g_{1}(x)=a_{1} x, g_{2}(y)=a_{2} y, h_{1}(x)=b_{1} x, h_{2}(y)=b_{2} y
$$

for some $a_{1}, a_{2}, b_{1}, b_{2} \in \mathbb{R}$. Now we have

$$
\begin{aligned}
f(z)=g(z)+i h(z) & =g(x)+g(i y)+i h(x)+i h(i y) \\
& =\left(a_{1}+i b_{1}\right) x+\left(a_{2}+i b_{2}\right) y \\
& =\alpha z+\beta \bar{z}
\end{aligned}
$$

for some $\alpha, \beta \in \mathbb{C}$. Now from the equation

$$
f(f(z))=f(z)-z
$$

we have for all $z \in \mathbb{C}$,

$$
\left(\alpha^{2}-\alpha+1+|\beta|^{2}\right) z=-\beta(\alpha+\bar{\alpha}-1) \bar{z},
$$

which implies

$$
\alpha^{2}-\alpha+1+|\beta|^{2}=-\beta(\alpha+\bar{\alpha}-1)=0 .
$$

Thus we have

$$
\alpha=\frac{1}{2} \pm i \sqrt{\frac{3}{4}+|\beta|^{2}} .
$$

This completes the proof.

\section{Acknowledgment}

This work was supported by Basic Science Research Program through the National Research Foundation of Korea(NRF) funded by the Ministry of Education, Science and Technology(MEST)(2012008507) 


\section{References}

[1] J. Aczél, J. Dhombres, Functional equations in several variables, Cambridge University Press, New York-Sydney, 1989.

[2] Nicole Brillouët-Belluot, On a symmetric functional equation in two variables, Aequationes Math. 68(2004), 10-20.

[3] M. E. Kuczma, On the mutual noncompatiablity of homogeneous analytic nonpower means, Aequationes Math. 45(1993), 300-321.

\section{Jae-Young Chung}

Department of Mathematics, Kunsan National University,

Kunsan 573-701, Korea.

E-mail: jychung@kunsan.ac.kr 